\title{
La protección del usuario en la domótica y las facilidades que ofrece
}

\section{User Protection in Home Automation and the Facilities It Offer}

\section{La protección del usuario}

José Luis Ortega Palacios

Carrera de Electrónica Informática del Instituto Superior Tecnológico, Ecuador. email:

joseluisortegapalacios@ outlook.com ORCID: https://orcid.org/0000-0003-1444-2849

Contacto: joseluisortegapalacios@outlook.com

Recibido: 18-02-2020 Aprobado: 26-03-2020

\section{Resumen}

Esta investigación se realizó con el objetivo de determinar la forma en que un usuario puede prevenir alguna emergencia en que un sistema sistema domótico llegue a fallar y se prolongue una situación en la cual debe de ser atendido, aquí están para eso los tecnólogos para prestar sus buenos servicios y darle mantenimiento a diarios para prevenir cualquier emergencia. Es por eso que está relacionada entre la automatización y el control inteligente a la vivienda que permite una función muy confortable como también el buen ahorro de energía eléctrica y una buena comunicación entre el usuario y el sistema, la domótica permite dar el conocimiento de muchos aparatos electrónicos como los sensores que recoge información proveniente que es capaz de captar, procesarla y después emitir una orden a los actuadores o salidas, permite dar respuestas a los requerimientos que plantean estos cambios sociales en cualquier diseño de casa o alguna edificación se puede dar un buen confort para el usuario que nos ayuda de muchas formas en lo que es iluminación, persianas, agua etc.

Palabras claves: Domótica, automatizar, edificación, dispositivos electrónicos, energía.

\section{Abstract}

Home automation is known to systems that are capable of automating either a dwelling or a building that is too large, which implements many circuits providing energy services, safe and very reliable in what is communication, which are integrated into the interiors and exteriors of a building can be wired or wireless. The objective of the investigation is to determine how a user can prevent any emergency in which the building system fails and a situation in which it must be attended is prolonged, here are the technologists for providing good services and giving daily maintenance to prevent any emergency.That is why it is related between automation and intelligent control of housing that allows a very comfortable function as well as good energy savings and good communication between the user and the system, home automation allows to give knowledge of many devices electronic as is the sensors that collects information that is able to capture, process it and then issue an order to what are actuators or outputs, allows to respond to the requirements posed by these social changes in any house design or any building can be give a good comfort for the user that helps us in many ways in what is lighting, blinds, water etc.

Keywords: Home, automation, automate, building, electronic devices, energy.

\section{Introducción}

El objetivo del proyecto es 'La Protección Del Usuario En La Domótica Y Las Facilidades Que Nos Ofrece', que forma parte de un sistema de automatización en casas y edificaciones inteligentes la cual se inició en Ecuador desde el 2008, el cual hubo un congreso y se hablo acerca de temas de la Domótica en la cual se refiere que las empresas ofrecen soluciones de sistemas hechos en el exterior y aquí solo se comercializa, pero en si la idea principal es no convertirnos consumidores sino en fabricadores de equipos para la Domótica porque el conocimiento es el mismo y como 
Ecuatorianos tenemos mucho potencial en desarrollar equipos tecnológicos (Bolton, 2008).

Aunque el ser humano aún no está muy entendido en las propiedades que ofrece la domótica es una realidad que en un lazo de tiempo no muy lejano estarán instalada en cada casa. ¿Pero que es la Domótica? Es un conjunto de tecnologías aplicadas en un hogar de manera que la convierte en una casa inteligente al ahorrar energía, como tener una buena seguridad y de muy bueno confortabilidad. (Millan, 2016).

La palabra Domótica proviene de la unión de las palabras domus (casa en latín) y tica (de automática), vocablo griego para decir 'que funciona por sí sola'.

La Domótica no tiene que ver nada con el confort, también abarca muchas cosas en diferentes tipos de áreas también se basa en el fundamento de un buen ahorro de energía y como no la protección al usuario en su bienestar en su buena atención de una casa automatizada que el usuario pueda descansar tranquilo. (Morales, 2012).

La domótica ha sido un buen factor en el ámbito tecnológico con el avance de las redes y telecomunicaciones que nos permite navegar y hablar de integración a nivel de esta red IP (Internet Protocol) es muy importante la parte electrónica para la automatización en casa y edificios inteligentes que ahora en este tiempo y a futuro nos ha facilitado de muchas formas en brindándonos una buena seguridad, ahorro de electricidad, y múltiples servicios gracias a la domótica nos permite innovar en diferentes áreas tecnológicas. (Gil Moreno, 2017)

Hay que tener muchas visiones más amplias en diferentes campos de trabajo todo esto lo podemos controlar mediante un celular o laptops de esto se basa la domótica en un conjunto de técnicas orientadas para hacer una automatización en un hogar o una edificación que está integrada en mucha tecnología en el bienestar del usurario brindando una buena protección y en las comunicaciones mediante cableados o inalámbricas con el fin de mejorar y tener una buena confortabilidad entre el usuario y la vivienda ofreciendo nuevos servicios mediante un sistema de control. (Quintero, 1999)

Los servicios principales de la domótica

Seguridad: Protege al usuario de muchas formas en lo que es sensor de humo en caso de incendios, alarmas personales en lo que es el uso doméstico como el gas el agua y el congelador que nos ayuda a visualizar que tanto contienen cada objeto como también se puede encender una alarma por falta de energía eléctrica.

Ahorro de Energía: Gestiona inteligentemente el ahorro de ahorro de energía en diferentes áreas de un hogar en los electrodomésticos, tales como televisores, aires acondicionados, el agua caliente, el riego, etc.

Comunicaciones: Mediante un buen control y supervisión remoto a la vivienda que lo podemos hacer a través de un celular o una computadora que tenga el acceso al internet. La instalación domótica permite comunicarse de diferentes formas como lo es la voz, también incluyendo texto, imágenes y hasta sonidos con redes locales como se conoce esta red de área local (LAN).

Confort: Convirtiendo en una vivienda más confortable para el usuario que sea de muy buen agrado haciendo diferentes actividades domésticas como: abrir, cerrar, encender, apagar, iluminación, climatización, persianas, electricidad, agua, gas entre otros. (Laserna, 2014)

Las casas han evolucionado de diversas funciones a lo largo de las décadas en el ámbito social como tanto en el área tecnológica en la década de los 80 cuando los locales empezaron a usar sistemas integrados para luego desarrollarse como usos domésticos en las casas urbanas y así se fue implementado este proyecto a nivel mundial, basándose en la innovación de la tecnología que tenia el usuario de una manera muy factible. (Rybczynski, 2008)

Es evidente que se viven en la actualidad tiempos finales, cambios sociales significativos promovidos por la nueva tecnología, estos cambios no solo se notan en el ámbito social y publico sino también en los privados es el impacto de las "modernas tecnologías de transmisión y de comunicación a distancia" que se caracteriza por la utilización de integración de tecnología a través del teléfono o mediante una Pc. (Echeverria, 2016)

El ambiente en una casa domótica hace que el usuario se sienta satisfecho que esto se lo conoce como un "ambiente tecnológico" que proporcionan espacios de ambientadores en casa, coche, oficina, etc. Esta claramente de que una casa domótica está hecha para una buena confortación en que la persona se sienta más cómoda, el buen ambiente hace que la persona tenga un buen confort (Eneo, 2013)

Muchos son los servicios que te pueden ofrecer en muchos lugares donde uno hace estas implementaciones de servicios electrónicos pero son 
pocos los que saben del buen funcionamiento y de la buena comodidad que es tener una casa de altamente tecnológica y que puede brindarte lo mejor para el usuario que este en buen ambiente y saber aplicar esto como una vida cotidiana. (SAKKAS, 2018)

El avance de las nuevas tecnologías de la información $\mathrm{y}$ las comunicaciones (TIC) se ha hecho de mucha tendencia en todos los ámbitos de la vida ya que ha hecho que en los últimos tiempos se haya hablado con mucha frecuencia sobre los edificios inteligente, áreas inteligentes, ciudades inteligentes y muchas oportunidades de negocio. Es bien palpable que el desarrollo en nuevas tecnologías está relacionado entre sí. (Clara, 2005)

El mundo ha cambiado bastante con el avance tecnológico que ha hecho en nosotros que veamos nuestro alrededor de una manera virtual en el que nos enfocamos en cada lugar que nos encontremos hay tecnología y como no las casas inteligente que han evolucionado mucho en país europeos como América latina y se puede ver hasta donde ha llegado y avanzara la tecnología. (ALTROCK, 2017)

Un conjunto de sistemas se encarga de regular y gestionar adecuadamente los elementos electrónicos y electrodomésticos incorporados en una vivienda como los aires acondicionados, las ventanas lo que es el suministro de agua para esto se necesita de un buen sistema que este permita hace todo en orden y con un buen funcionamiento para controlar y automatizar la vivienda. (Oberto, 2006)

Te da un buen ahorro y control de energía eléctrica facilitándote un consumo bajo un claro ejemplo seria la calefacción que en ves de dejarla encendida todo el día puedes programar por un lazo de tiempo de minutos, aparte otra ventaja es la seguridad puede detectar cualquier intruso que en tu casa quiera robarte o una fuga de gas o también un escape de agua todo esto más el confort para hace una casa de alta gama y muy cómoda. (Colina, 2019)

Un sistema inteligente hace que muchas personas le sean muy fácil de usar solo con un dispositivo que ahora en la actualidad le llamamos teléfonos móvil que conectado a un red de internet inalámbrica podemos hacer muchos quehaceres de diferentes tipos y uno estar a distancia pude dar mandos a distintos dispositivos, estamos viviendo en una era tecnológica donde hay altos niveles de automatización. (Peréz, 2010)

Hay muchas casas las cuales tiene muchos beneficios unas de ellas es la instalación
En el mundo que vivimos tecnológicamente existen distintos estándares domóticos para hacer y conformar un hogar digital hay diferentes tipos de estándares que nos permite según modificar y hacer una casa como nosotros queramos pero el problema es que si utilizamos un estándar para una sola vivienda tiene que ser determinado para una vivienda se obliga adquirir todos los dispositivos para una sola vivienda esto quiere decir que cada vivienda según la estructura debe ser compatible. (Camps, 2006)

La Domótica no afecta en nada mas bien ayuda mucho a todo tipo de personas desde los niños hasta los ancianos porque es ofrecer un buen servicio de seguridad en el que nosotros podamos ver lo grandioso que es ver una casa inteligente con todos los temas y medidas que pueda ayudarnos en un futuro cercano de mucha seguridad y poder hacer un hogar de mucha productividad. (HOKENSON, 2015)

Muchas veces el hombre ha intentado vivir en un habitad confortable hasta que se inició la domótica que ha modificado su entorno físico y que puede variar mucho más en el tiempo y que esto ha evolucionado en tan poco tiempo donde ya hay casas que están lleno de muchos sensores y que puede varias muchas cosas en la casa pero utilizan los mismos dispositivos y son muy flexibles y adaptables. (Andrade, 2001)

Existen desventajas en las casas inteligentes uno de estos es invertir es muy costoso y son precios muy elevados para el usuario ya que son sistemas automatizados y aparte también es que hay que hacer mantenimientos a estos equipos los cuales son muy complejos y costosos y otra desventaja es la interferencia de datos la velocidad del internet dependiendo de cuantos puntos estén conectados siempre se conectan en forma de anillo. (Maya, 2004)

En la actualidad hay muchos sistemas domóticos que están integrados en la informática y muchas tecnologías de la información en el cual se espera una casa con un buen confort, seguridad para nuestra familia que este activa para cualquier caso de emergencia y por qué no un buen ambiente en el cual la casa habilita sus mejores servicios con la ayuda de la programación. (Graziani, 2018)

\section{Objetivo General:}

Dar a conocer como se ha actualizado la domótica desde la décadas de los 70 cómo ha evolucionado con el paso del tiempo y saber lo importante que es ahora en la actualidad como podemos hacer un buen ahorro de energía solo implementando estos circuitos electrónicos en las viviendas y edificios, aparte de eso 
nos permite tener una buena seguridad tanto para el usuario como para la familia solo implementando cámaras de seguridad y cercas eléctricas y porque no los famosos sensores de fuga de gas, escape de agua y expansores de agua en caso de un conato de incendio.

Desarrollar la implementación de la domótica para tener un buen confort de nuestras áreas de la casa como los aires climatizados, calefacción, iluminación entre otros es muy importante saber cómo ahora las nuevas tecnologías han evolucionado tanto que todo lo podemos hacer con programaciones y dar órdenes a nuestra casa mediante un teléfono móvil que este conectados a una red inalámbrica de internet, es ver la maravilla que podemos hacer ahora en este siglo conocido como un mundo tecnológico.

\section{Objetivos Específicos:}

- Brindar una buena seguridad al usuario y que este se sienta muy confort con la estructura y diseño de una casa inteligente.

- Dar orientación a los usuarios sobre la domótica que puede ayudarnos en distintas áreas donde nos encontremos mediante programaciones.

- Reconocer cada implementación de los circuitos electrónicos que están instalados en una casa para ofrecer buen confort, seguridades externas e internas.

\section{Materiales y Métodos}

El trabajo investigativo se empezó a realizar el 5 de Noviembre del 2019 y se concluyo en el 08 de Marzo de 2020, cuyo proyecto se lo investigo en el Instituto Superior Tecnológico Portoviejo como también se lo realizo en áreas que tienen implementando estos circuitos electrónicos como: Los edificios del estado, hospitales, edificios del comité de emergencias a Nivel Nacional aquí entra; el Cuerpo de Bomberos, el ECU 911, Policía Nacional, etc.

En los materiales esta basado en el Arduino que un microcontrolador que esto se ha hecho en distintas casas para poder automatizarlas y dar mandos a los sensores, luces, puertas, ventanas y cuartos automatizados.

También se desarrollo una encuesta tanto para la población como para estudiantes universitarios para tener como objetivos que tan importante es la domótica tanto en la ciudad como a nivel nacional y en que puede facilitarnos o que seguridad nos puede dar como resultado implementar circuitos electrónicos en nuestras casas o edificios, es bien claro que la domótica va mas allá de una casa inteligente porque facilita realizar distintas actividades mientras no estemos dentro de ella, cada persona que llenaban esta encuesta le gustaba la idea de implementar sensores, cámaras de seguridades y focos que con un solo movimiento se encendieran se realizo esta encuesta con el fin de ver las perspectivas que tienen las personas al escuchar de una casa domótica.

Esta encuesta reflejaba las ideas como usuarios planean tener una casa totalmente automatizada y en breve mostraremos los resultados de cada pregunta realizada en la encuesta.

\section{Resultados}

\begin{tabular}{|l|l|l|l|}
\hline Orden & Alternativa & Frecuencia & Porciento \\
\hline A & Siempre & 20 & 50,00 \\
\hline B & A veces & 10 & 25,00 \\
\hline C & Rara vez & 5 & 12,50 \\
\hline D & Nunca & 5 & 12,50 \\
\hline Total & & 40 & 100 \\
\hline
\end{tabular}

Tabla 1; ¿Usted ha visitado una casa domótica? Fuente: Universidad Técnica De Manabí

Una casa inteligente causa a las personas un gran impacto en lo que es una buena automatización y también como protección hacia la persona en lo que refleja la tabla 1, la mayoría de las personas encuestada han visto o visitan una casa o edificio de manera inteligente o es una manera muy eficaz de que esto evolucione mucho más a futuro en lo que crea un gran impresión en los demás, cabe recalcar que estas implementaciones sirven de mucho en cualquier casa que estén bien estructurada siempre y cuando exista un buen confort y eficacia para el usuario para protección en cualquier emergencia.

\begin{tabular}{|c|c|c|c|}
\hline Orden & Alternativa & Frecuencia & Porciento \\
\hline A & Todos & 10 & 25,00 \\
\hline B & Algunos & 20 & 50,00 \\
\hline $\mathrm{C}$ & Ninguno & 10 & 25,00 \\
\hline \multicolumn{2}{|l|}{ Total } & 40 & 100 \\
\hline
\end{tabular}

Tabla 2; ¿Todas las edificaciones que ha visitado o conoce tienen un buen parqueo?

Fuente: Universidad Técnica De Manabí

En toda casa inteligente o casa debe existir un buen parqueo para así tener más espacio y conformidad como una buena señalización en caso de emergencia, los resultados que refleja la tabla 2 es que las personas que realizaron la encuesta muchas personas dieron opiniones que sería de mucha utilidad tener un buen 
parque el cual sea programado con la finalidad que el usuaria apenas llegue a casa el parqueadero se vaya abriendo lo cual ahorre tiempo y tenga una buena seguridad, ya que son pocos los que tienen un sistema así.

\begin{tabular}{|l|l|l|l|}
\hline Orden & Alternativa & Frecuencia & Porciento \\
\hline A & Mucho & 30 & 75,00 \\
\hline B & Poco & 5 & 12,50 \\
\hline C & Nada & 5 & 12,50 \\
\hline Total & & 40 & 100 \\
\hline
\end{tabular}

Tabla 3; ¿Le parece bien la implementación de circuitos electrónicos en las casas?

Fuente: Universidad Técnica De Manabí

Una implementación de circuitos electrónicos en una casa es de muy buena opción ya que esto nos facilita mucho siendo de un pc o un celular inteligente lo que permite hacer muchas actividades domésticas, en la tabla 3 refleja que muchas personas están de acuerdo con esta gran implementación que nos da facilidad a diferentes tipos de actividades en una casa o edificio inteligente que a través de esto nos brinda seguridad, confortabilidad y un ahorro de energía.

\begin{tabular}{|l|l|l|l|}
\hline Orden & Alternativa & Frecuencia & Porciento \\
\hline A & Mucho & 13 & 32.5 \\
\hline B & Poco & 20 & 50,00 \\
\hline C & Nada & 7 & 17,50 \\
\hline Total & & 40 & 100 \\
\hline
\end{tabular}

Tabla 4; ¿Le interesa mucho los temas de un edificio inteligente en la ciudad?

Fuente: Universidad Técnica De Manabí

Los edificios inteligentes son muy importantes porque estos están diseñados y estructurados de manera muy segura en todo tipo de emergencia están sensorizados ya sea en sus puertas, pasillos, y en diferentes áreas en contra incendio, sismos y en robo, en la tabla 5 las personas que realizaron la encuesta hablaron que es bueno implementar esos circuitos en todos los edificios y no solo lo del gobierno, este sistema automatizado ayudara a proteger a la persona de todo peligro es importante el bienestar de uno con una buen conformidad y que el usuario se relacione con las cosas electrónicas que es innovador a nivel mundial.

\begin{tabular}{|l|l|l|l|}
\hline Orden & Alternativa & Frecuencia & Porciento \\
\hline A & Mucho & 20 & 50,00 \\
\hline B & Poco & 13 & 32,50 \\
\hline C & Nada & 7 & 17,50 \\
\hline Total & & 40 & 100 \\
\hline
\end{tabular}

Tabla 5; ¿Hay muchos edificios o casas en su ciudad de manera inteligente?

Fuente: Universidad Técnica De Manabí

Los edificios o casas domóticas han sido de mucho uso en la que el usuario puede darse cuenta que la tecnología día a día avanza con ayuda de un circuito electrónico uno puede ver más allá lo que puede realizar en la tabla 6 como resultados no da a ver que hay variedades de casas que usan al menos 3 o 4 circuitos de manera inteligente el ya utilizando una cámara de seguridad o un cerco eléctrico o porque no un garaje electrónico ya es una casa de manera inteligente.

\begin{tabular}{|l|l|l|l|}
\hline Orden & Alternativa & Frecuencia & Porciento \\
\hline A & Siempre & 12 & 30,00 \\
\hline B & A veces & 8 & 20,00 \\
\hline C & Rara vez & 9 & 22,50 \\
\hline D & Nunca & 11 & 27,50 \\
\hline Total & 40 & 100 \\
\hline
\end{tabular}

Tabla 6; ¿Ha visto de estas características con sensores y cámaras las casas?

Fuente: Universidad Técnica De Manabí

En ciertas casa y edificaciones hay estos sistemas de sensores pero en la mayoría hay estos sistemas el cual brinda seguridad y conformidad al usuario en que forma seguridad porque al implementar estas cámaras están activadas con una alarma y cualquier cosa que haga poner en riesgo al usuario mediante un móvil la puede activar y lo que es sensor ya sea tener una puerta que con el movimiento de una persona se abra y porque no las ventanas que tiene su persiana eléctrica, en la tabla 7 muchas personas han visto como otras no, edificaciones o casas con estas características, pero si dieron muchas opiniones que es bueno implementar esto en todas las casa.

\begin{tabular}{|l|l|l|l|}
\hline Orden & Alternativa & Frecuencia & Porciento \\
\hline $\mathrm{A}$ & Excelente & 28 & 70,00 \\
\hline $\mathrm{B}$ & $\begin{array}{l}\text { Muy } \\
\text { bueno }\end{array}$ & 10 & 25,00 \\
\hline $\mathrm{C}$ & Bueno & 1 & 2,50 \\
\hline $\mathrm{D}$ & Regular & 1 & 2,50 \\
\hline $\mathrm{E}$ & Deficiente & 0 & 0,00 \\
\hline Total & & 40 & 100 \\
\hline
\end{tabular}

Tabla 7 ¿Está de acuerdo con esta implementación en su ciudad para brindar una buena seguridad?

Fuente: Universidad Técnica de Manabí

Esta implementación en el tema de la Domótica es un factor muy amplio, pero de alta eficacia y sobre todo de 
muy poca demanda ya que en ciertas ciudades no tiene mucho conocimiento de esto, pero es bueno para que las personas conozcan de esto, la tabla 7 la mayoría de personas estuvo de acuerdo a esto para tener una ciudad segura y de manera inteligente que nos permite hacer muchas actividades rutinas diarias mientras tenemos una ciudad segura.

Una casa totalmente inteligente con muchos dispositivos electrónicos nos permite que nuestra vida se mas fácil porque nosotros seriamos los que hiciéramos mando mediante un celular inteligente, la tabla 8 nos refleja como resultados que la mayoría quisieran una casa inteligente por lo seguro, conforme, ahorro de energía, y por qué es con redes inalámbricas.

\begin{tabular}{|l|l|l|l|}
\hline Orden & Alternativa & Frecuencia & Porciento \\
\hline A & Excelente & 30 & $75,00 \%$ \\
\hline B & $\begin{array}{l}\text { Muy } \\
\text { bueno }\end{array}$ & 6 & $15,00 \%$ \\
\hline C & Bueno & 3 & $7,50 \%$ \\
\hline D & Regular & 1 & $2,50 \%$ \\
\hline E & Deficiente & 0 & $0,00 \%$ \\
\hline Total & 40 & 100 & \\
\hline
\end{tabular}

Tabla 8; ¿Qué tal seria que usted tuviera una casa inteligente?

Fuente: Universidad Técnica De Manabí

La encuesta para muchas personas le resulto muy interesante por parte de las personas en implementar circuitos electrónicos en las estructuras sean casas o edificios inteligentes ya que esto ha evolucionado de manera inteligente que hasta en las casas podemos ver no muchos aparatos electrónicos, pero si la mayoría los implementan no completo, pero si a poco a poco por el tema que es muy costoso.

\section{Discusión}

En la Domótica hay muchos factores en el cual hay sus ventajas y desventajas una de las ventajas en las viviendas inteligentes que es que te ofrecen una buena confortabilidad y tiene sus sistemas muy seguros, pero una de sus desventajas es que son muy costosos tener una implementación completa en la casa ya que son muchos sistemas programados y requieren tanto de un buen internet como de componentes que son caros y se debe tener en cuenta que hay diferentes tipos de circuitos.
Los nuevos conocimientos del proyecto son que hay partes en que la domótica están integrados circuitos de otros niveles que son mas extensivos y esto en ocasiones de fallar son muy complicados de repararlos en los resultados recalca que es de gran ayuda una casa domóticas en todos los ámbitos lo único malo sería el precio adquirir y hacer una de estas pero de ofrecerte los mejores servicios que puede brindarte una casa

\section{Conclusiones}

Es necesario la implementación de los circuitos electrónicos en las viviendas y edificio ya que ahora vienen automatizadas y programadas para el usuario el que uno se siente seguro de convivir por que esta rodeada de sin números de sensores como lo es sensores de movimientos, de focos, puertas y en los aires acondicionados que están programados por cierto límite de tiempo para hacer un enfriamiento es necesario que los usuarios estén enterado de la buena seguridad en que nos pude ayudar en diferentes áreas mientras uno se encuentra fuera de casa mediante un teléfono móvil puede observar que es lo que sucede dentro de la vivienda es necesario reconocer que todos estos circuitos trabajan conectadas en una red inalámbricas ya vienen programada por un lapso de tiempo son de mucha eficacia ya que todo esto implementado en las viviendas ofrecen un buen ahorro de energía en la que nos facilita mucho en los gastos y una casa esta conformada por un buena estructura antisísmica y en todo caso de emergencia cualquier fuga de gas estos sensores lo detectaran y han su función así mismo en la noche todas las puertas se activan los seguros y hasta con reconocimiento de voz uno puede dar una señal.

\section{Bibliografías}

AlLTROCK, J. A. (2009-2010). Domótica e Inmótica. Obtenido de Domótica e Inmótica: http://www.nebrija.es/ jmaestro/ATA018/Dom otica.pdf

Andrade, M. (2001). Departamento de Tecnología de la Edificación. Madrid. Simulación automatizada de la iluminación arquitectónica en fachadas. Facultad de Arquitectura y Diseño. Universidad del Zulia.

Bolton, W., \& Ramírez, F. J. R. (2001). Ingeniería de control (pp. 1-3). Mexico: Alfaomega.

Camps, R. D., Yagüe, J. L. P., Luján, J. L. P., Blasco, P. P., \& Escribá, J. C. C. (2006). Arquitectura SCHome: Acceso remoto al hogar digital. Actas 
de las XXVII Jornadas de Automática (JA2006), 348-354.

Clara (2005) LSB Tecnologia Inteligente. Recuperado el 04 de 10 de 2016, de X1O Sistema como Funciona:

http://www.lsb.es/imagenes/x10_introduccion. pdf

Colina Mendoza \& Cristóbal Romero Morales, F. J. (2007). Domótica e Inmótica. Viviendas y edificios inteligentes. 2 Edición. México: Alfaomega grupo Editor. Cubero, A. C. (09 de 2008). Biblioteca Universidad de Sevilla. Obtenido de Biblioteca Universidad de Sevilla

Echeverría, J. (1995). Cosmopolitas domésticos. Barcelona: Anagrama. Heidelberg, pp. 227-246.

Éneo Ramírez. (2007). Edificio Mushuc Runa para Noviembre en la cuidad de Quito. La Hora. Obtenido

de http://www.lahora.com.ec/index.php/noticias/s how/601253/-

Graziani G, 2018, Descripción del Prototipo de una Vivienda Bioclimática Con Enfoques Domóticos Aplicando VRML, Vol. 1 pp 2-3.

Lasserna. (2014). SENSOR NETWORK. 2003, de ARCHITECTURES PROTOCOLS Sitio web: https://bibdigital.epn.edu.ec/bitstream/15000/9 38/1/CD-1838\%282009-01-21-12-2509\%29.pdf

Luis Hokenson Álvarez, A. M. (16 de Junio de 2007). UNIVERDSIDAD DE OVIEDO. Obtenido de REPOSITORIO UNIVERDSIDAD DE OVIEDO.

Maya, 2004 Karimanal, A. E. (02 de 2012). Revista Española de Electrónica. Obtenido de Revista Española de Electrónica: http://www.redeweb.com/_txt/687/p56.pdf

Kemisa. (s.f.). kemisa. Recuperado el 03 de octubre de 2017, de kemisa: http://www.kemisa.es/es/circuitos-paraordenador/42-encendidoautomatico-del-pc.html

Millan \& Carlos. (2016). APRENDA RAPIDAMENTE A PROGRAMAR. 2006, de DOMÓTICA, Vol. 1 pp 3-4.

Morales Edison, F., González, V. M., Poo, R., García, M., \& Olaiz, R. (2010). Design and Development of An Automatic Small-Scale
House For Teaching Domotics. Proceedings of the Frontiers in Education Conference, 2010. On 31st Annual - Volume 01. Washington, DC, USA: IEEE Computer Society, pp T3c-1-5.

Moreno Gil, J., Lasso Tarraga, D., \& Rodríguez Dieguez, E. (2017). Instalaciones automatizadas en viviendas y edificios (1st ed.). Madrid: Paraninfo Cengage Learning.

Oberto Palacios \& Manuel Cabello, M. S. (2016). Circuitos eléctricos básicos II (Instalaciones eléctricas interiores). Editex.

Pérez David. Domótica Viva. (08 de Noviembre de 2012). Domótica Viva. Obtenido de Domótica Viva: http://www.domoticaviva.com/X-10/X10.htm Flores, J. A. (marzo de 2007). Repositorio Escuela Politécnica Nacional. Obtenido de Repositorio Escuela Politécnica Nacional: http://bibdigital.epn.edu.ec/bitstream/15000/39 1/1/CD-0798.pdf

Quintero González, J. M., Lamas Graziani, J., \& Sandoval Gonzáles, J. D. (1999). Sistemas de control para viviendas y edificios: domótica.

Rybczynski, W. (2008). Instalaciones automatizadas en viviendas y edificios (2nd ed.). Buenos aires: Emece Editores.

Sakkas. (22 de Mayo de 2018). Edificios inteligentes se toman Quito. La Hora. Obtenido de http://www.lahora.com.ec/index.php/noticias/s how/1038174/1/Edificios_inteligentes_se_toma n_Quito.html\#.VDtOT_15N7k LSB. (s.f.). 